\title{
Lithium-Assisted Electrochemical Welding in Silicon Nanowire Battery Electrodes
}

\author{
Khim Karki, ${ }^{\dagger}$ Eric Epstein, ${ }^{\dagger}$ Jeong-Hyun Cho, ${ }^{\ddagger}$ Zheng Jia, ${ }^{\S}$ Teng Li, ${ }^{\S}$ S. Tom Picraux, ${ }^{\ddagger}$ \\ Chunsheng Wang, ${ }^{*}, l$ and John Cumings, ${ }^{* \dagger}$ \\ ${ }^{\dagger}$ Department of Materials Science and Engineering, University of Maryland, College Park, Maryland 20742, United States \\ ${ }^{\ddagger}$ Center for Integrated Nanotechnologies, Los Alamos National Laboratory, Los Alamos, New Mexico 87545, United States \\ ${ }^{\S}$ Department of Mechanical Engineering and "Department of Chemical and Biomolecular Engineering, University of Maryland, \\ College Park, Maryland 20742, United States
}

\section{Supporting Information}

ABSTRACT: From in situ transmission electron microscopy (TEM) observations, we present direct evidence of lithiumassisted welding between physically contacted silicon nanowires (SiNWs) induced by electrochemical lithiation and delithiation. This electrochemical weld between two SiNWs demonstrates facile transport of lithium ions and electrons across the interface. From our in situ observations, we estimate the shear strength of the welded region after delithiation to be approximately $200 \mathrm{MPa}$, indicating that a strong bond is formed at the junction of two SiNWs. This welding phenomenon could help address the issue of capacity fade in nanostructured silicon battery electrodes, which is typically caused by fracture and detachment of active materials from the current collector. The process could provide for more robust battery performance either through self-healing of fractured components that remain in contact or through the formation of a multiconnected network architecture.

KEYWORDS: Silicon nanowires, welding, self-healing, interfacial lithium diffusivity, in situ TEM, lithium-ion battery

$\mathrm{S}^{\mathrm{i}}$ ilicon is an auspicious candidate to replace today's widely utilized graphitic anodes in lithium ion batteries because its specific energy capacity $(3579 \mathrm{mAh} / \mathrm{g})$ is nearly 10 times that of carbon $(372 \mathrm{mAh} / \mathrm{g})$ and because it is an inexpensive and abundant resource. ${ }^{1-7}$ However, during lithiation and delithiation, Si undergoes a massive volume change ( $2300 \%)$, which has been reported to cause fracture and pulverization of the electrode, thereby leading to capacity degradation and failure of the battery cell. ${ }^{8-12}$ To circumvent the problem, researchers have developed nanostructured $\mathrm{Si}$ electrodes using thin films, ${ }^{13}$ nanoparticles, ${ }^{14}$ nanowires, ${ }^{6}$ and nanotubes. ${ }^{15,16}$ Compared to bulk forms, nanostructured $\mathrm{Si}$ exhibits higher yield strength and fracture strength and is better capable of accommodating massive volume strains. ${ }^{17,18}$ Despite these benefits, capacity fade during cycling remains a critical issue even for nanostructured $\mathrm{Si}$ electrodes, presenting challenges for their commercial development.

Cracking and pulverization are known to be mitigated in part by nanostructured forms of silicon, but several recent studies indicate that cracking can still occur in nanostructures subject to a variety of conditions. ${ }^{19-22}$ In contrast to this detrimental effect, we report an opposite effect, which may enable selfhealing nanostructured Si-electrodes. While performing in situ transmission electron microscopy (TEM), we observe that contacted surfaces of otherwise physically distinct silicon nanowires are fused together after lithiation and delithiation. To date, there have only been a few post-mortem studies suggesting the occurrence of bonding and coalescence of nanomaterials in lithium battery electrodes. ${ }^{23-27}$ For example, $\mathrm{Li}$ et al. ${ }^{24}$ report the coalescence of Si nanoparticles from TEM images of cycled and dismantled composite electrodes. However, direct evidence and a detailed understanding of the process are both still lacking. We here report in situ TEM observations of the welding of physically contacted silicon nanowires (SiNWs) during electrochemical lithiation and delithiation. Our data indicates that this welding phenomenon is predominantly an electrochemically induced process, occurring at room temperature. Moreover, we demonstrate that the weld formed between SiNW surfaces is mechanically robust and exhibits evidence of facile transport of lithium ions, which are both desirable properties for enhanced battery performance.

The SiNWs used in this study (typically with $\langle 211\rangle$ growth direction) are synthesized directly on TEM grids using bottom-

Received: November 17, 2011

Revised: January 30, 2012

Published: February 16, 2012 
up chemical vapor deposition (CVD) via the vapor-liquidsolid (VLS) process (see Supporting Information, Figure S1). The in situ operation and characterization is employed using a JEOL JEM-2100 $\mathrm{LaB}_{6}$ TEM. We use a specialized piezoactuated NanoFactory holder capable of three-dimensional coarse motion with subangstrom accuracy and simultaneous ability to image during manipulation (Figure 1a). The

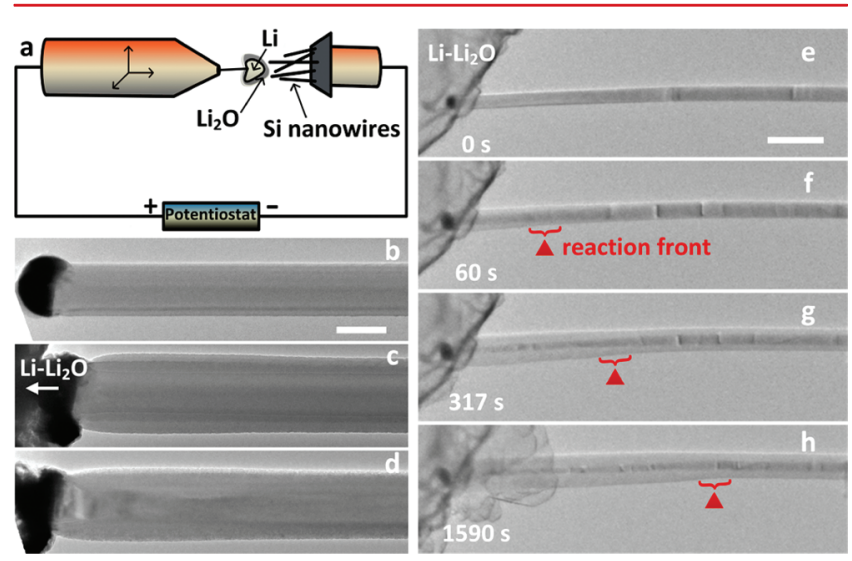

Figure 1. In situ lithiation and delithiation of silicon nanowires in TEM. (a) Schematic of experimental setup using lithium metal as a counter electrode. (b) A pristine nanowire approaching a lithium metal source. Scale bar of $200 \mathrm{~nm}$. (c) Volume expansion as seen after lithiation for $30 \mathrm{~min}$ with a potential of $-4 \mathrm{~V}$ applied to silicon nanowire versus the lithium electrode. (d) Volume contraction of nanowire after delithiation for $30 \mathrm{~min}$ with applied potential $+4 \mathrm{~V}$ vs lithium. $(\mathrm{e}-\mathrm{h})$ Lithiation propagation front observed as a function of time in another SiNW. Scale bar of $500 \mathrm{~nm}$.

manipulation tip carries a piece of lithium metal, which acts simultaneously as a $\mathrm{Li}$ source, a reference electrode, and a counter electrode, while a thin layer of native $\mathrm{Li}_{2} \mathrm{O}$ due to the residual vacuum of the microscope serves as a solid electrolyte, allowing the diffusion of $\mathrm{Li}^{+}$between the electrodes under potential bias. $^{22,28,29}$ Figure $1 \mathrm{~b}$ shows a pristine crystalline $\mathrm{SiNW}$ of diameter $212 \mathrm{~nm}$ adjacent to the approaching $\mathrm{Li}_{2} \mathrm{O}$ coated $\mathrm{Li}$ source. Figure 1c shows that partial lithiation occurs when a potential of $-4 \mathrm{~V}$ is applied to the SiNW with respect to lithium metal. Upon lithiation, the increase in diameter of the nanowire is observed to be $50 \%(318 \mathrm{~nm})$. Upon delithiation, with an applied bias of $+4 \mathrm{~V}$ versus lithium metal, the nanowire shrinks to a diameter of $278 \mathrm{~nm}$ (Figure 1d). Because of large volume and structural changes during lithiation and delithiation, the reaction front can be readily identified from in situ TEM images as it traverses along the length of a NW (Figure 1e-h). Therefore, relative Li transport rates within various sections of a SiNW during lithiation and delithiation can be estimated from the speed of the reaction front, as determined by a series of TEM images captured during lithiation and delithiation.

To investigate $\mathrm{Li}$ transport across contacted SiNWs, two separate nanowires are brought into contact with each other by bending one of them with the manipulator probe at $0 \mathrm{~V}$ bias, as shown in Figure 2. One nanowire with a diameter of $143 \mathrm{~nm}$ is contacted by the lithium source while the other with diameter of $98 \mathrm{~nm}$ remains isolated (Figure 2a,d), contacted only indirectly, via the larger nanowire. Both nanowires are connected at the base to the silicon growth substrate. When a potential of $-3 \mathrm{~V}$ with respect to lithium metal is applied to the silicon substrate, lithiation proceeds in the nanowire

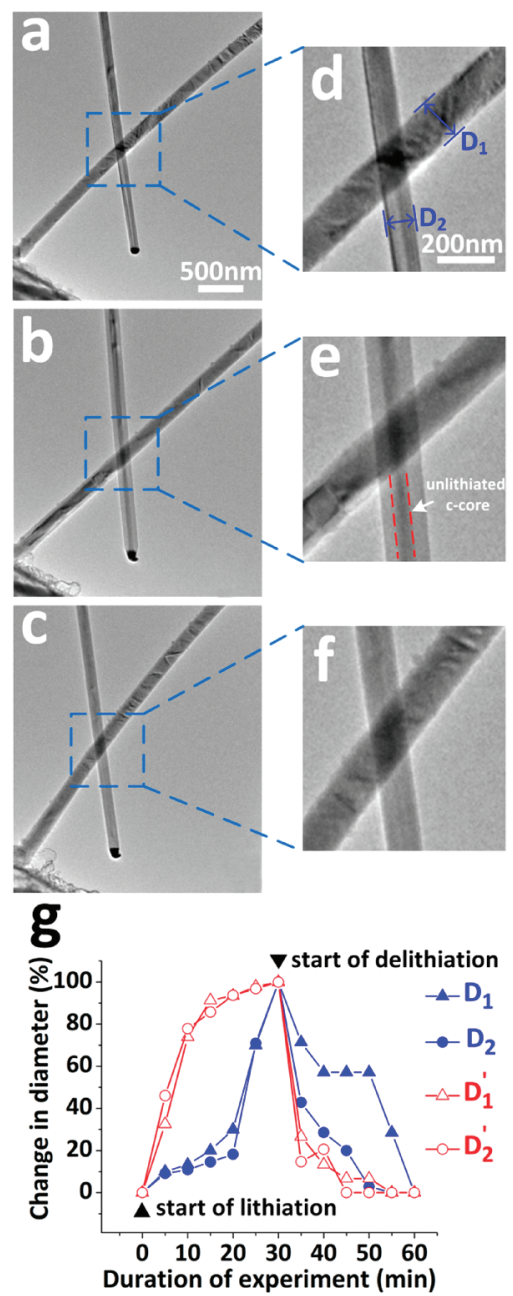

Figure 2. Lithiation and delithiation observed in crossed silicon nanowires. (a) Pristine nanowires. (b) Swelling of both nanowires after lithiation. (c) With delithiation, both nanowires contract, however, and fail to reach the original volume. (d-f) Blown up images focusing on crossed part of nanowire representing panels a-c, respectively. The measured $D_{1}$ and $D_{2}$ diameters for $d-f$ are $(143,98)$ $\mathrm{nm},(167,148) \mathrm{nm}$, and $(161,125) \mathrm{nm}$ respectively. (g) Changes in diameter in the $\mathrm{D}_{1}$ and $\mathrm{D}_{2}$ section of the crossed SiNWs (Figure $2 \mathrm{~d}$ ) plotted with respect to duration of experiment. Also observed results are plotted for another set of crossed SiNWs (Figure S2, Supporting Information) denoted as $\mathrm{D}_{1}^{\prime}$ and $\mathrm{D}_{2}^{\prime}$.

directly contacting the $\mathrm{Li}_{2} \mathrm{O}$, propagating in both the radial and axial directions (Figure 2b,e). At the same time, we also observe lithiation advancing in the crossed nanowire, which is not itself contacting the lithium source. To test $\mathrm{Li}$ transport across the resulting interface of the two SiNWs, the diameter changes were subsequently monitored during delithiation by supplying the Si substrate with a bias of $+3 \mathrm{~V}$ versus the lithium metal source (Figure 2c,f). As delithiation progresses, both nanowires are observed to decrease in diameter. We controlled the lithiation and delithiation times to make sure that the reaction front in the primary SiNW proceeded past the contact point of the two nanowires but did not reach to the Si substrate. Adjacent nanowires that are not in contact with the lithiated nanowires show no lithiation behavior, also confirming that the lithium transport is indeed through the SiNW-SiNW contact and not, for example, mediated via the Si substrate. 
To test for any possible delay due to diffusion across the welded interface, we quantify the relative diameter changes of directly contacted and indirectly contacted nanowires during lithiation and delithiation, as shown in Figure $2 \mathrm{~g}$. These experiments were conducted by applying the bias voltages and observing the nanowire evolution at video rates for approximately $15 \mathrm{~s}$, periodically every five minutes during lithiation. At times between observations, the illuminating electron beam is blanked in order to avoid possible beam effects on the sample. We define the variable diameters of the directly contacted and indirectly contacted nanowires in the vicinity of the junction as $D_{1}$ and $D_{2}$, respectively, as shown in Figure $2 d$. We also define a corresponding pair of diameters, $\mathrm{D}_{1}{ }^{\prime}$ and $\mathrm{D}_{2}{ }^{\prime}$, for a second pair of nanowires shown in Supporting Information Figure $S 2$. Table 1 shows the diameters $D_{1}, D_{2}$,

Table 1. Diameters of Two Sets of Crossed SiNWs in the Vicinity of the Crossing in Their Pristine, Lithiated, and Delithiated States

\begin{tabular}{cccc} 
SiNW diameter & $\begin{array}{c}\text { pristine } \\
\left(D_{\mathrm{P}}, \mathrm{nm}\right)\end{array}$ & $\begin{array}{c}\text { lithiated } \\
\left(D_{\mathrm{L}}, \mathrm{nm}\right)\end{array}$ & $\begin{array}{c}\text { delithiated } \\
\left(D_{\mathrm{D}}, \mathrm{nm}\right)\end{array}$ \\
$\mathrm{D}_{1}$ & 143 & 173 & 159 \\
$\mathrm{D}_{2}$ & 98 & 153 & 118 \\
$\mathrm{D}_{1}{ }^{\prime}$ & 108 & 154 & 139 \\
$\mathrm{D}_{2}{ }^{\prime}$ & 142 & 205 & 171 \\
\hline
\end{tabular}

$\mathrm{D}_{1}{ }^{\prime}$, and $\mathrm{D}_{2}{ }^{\prime}$ of each respective $\mathrm{SiNW}$ in their pristine, lithiated, and delithiated states. We calculate fractional diameter changes during the lithiation phase according to the ratio $\left(D-D_{\mathrm{P}}\right)$ / $\left(D_{L}-D_{\mathrm{P}}\right)$, and during the delithiation phase according to $(D-$ $\left.D_{\mathrm{D}}\right) /\left(D_{\mathrm{L}}-D_{\mathrm{D}}\right)$, where in both expressions, $D_{\mathrm{P}}, D_{\mathrm{L}}$, and $D_{\mathrm{D}}$ correspond to the diameter of each SiNW in its pristine, lithiated, and delithiated state, respectively, and $D$ represents the measured diameter as it varies with time. Using these expressions, we report the relative change in diameter of contacted nanowires as a function of time during lithiation and delithiation in Figure $2 \mathrm{~g}$. These measurements were obtained from experiments, during which we lithiated each crossedSiNW system for $30 \mathrm{~min}$, and subsequently delithiated for 30 min. Using the incremental diameter changes of each nanowire, we qualitatively compare the lithium propagation rates among SiNWs. With the exception of a delayed contraction briefly exhibited by $\mathrm{D}_{2}$ upon delithiation, we observe that the rates of $\mathrm{Li}$ propagation in the indirectly contacted SiNWs are identical to those in the directly contacted SiNWs. Here, we note that SiNW system $\mathrm{D}_{1}{ }^{\prime}-\mathrm{D}_{2}{ }^{\prime}$ lithiates faster and with less time-delay than SiNW system $D_{1}-D_{2}$. This difference in electrochemical behavior may be attributed to variable response of the $\mathrm{Li}_{2} \mathrm{O}$ electrolyte to the applied potential between separate experiments. ${ }^{30}$ We may also attribute the delayed contraction of $\mathrm{D}_{2}$ to variant behavior of $\mathrm{Li}_{2} \mathrm{O}$ or, alternatively, to different mechanical behavior of the larger nanowire $\left(D_{1}\right.$, e.g., increased stress and cracking) as compared to the smaller nanowire $\left(D_{2}\right)$. Despite these experimental variations, the curves of Figure $2 \mathrm{~g}$ provide evidence for identical rates of $\mathrm{Li}$ propagation in indirectly contacted SiNWs to those in SiNWs directly contacting the $\mathrm{Li}$ source. Therefore, we conclude that there is no additional barrier to $\mathrm{Li}$ diffusion between physically contacted SiNW surfaces at the charging rates used for these studies (approximately 2C, a rate which corresponds to charging or discharging in 0.5 hours).
We investigate the strength of the weld formed between two SiNWs using an experimental procedure shown in Figure 3.

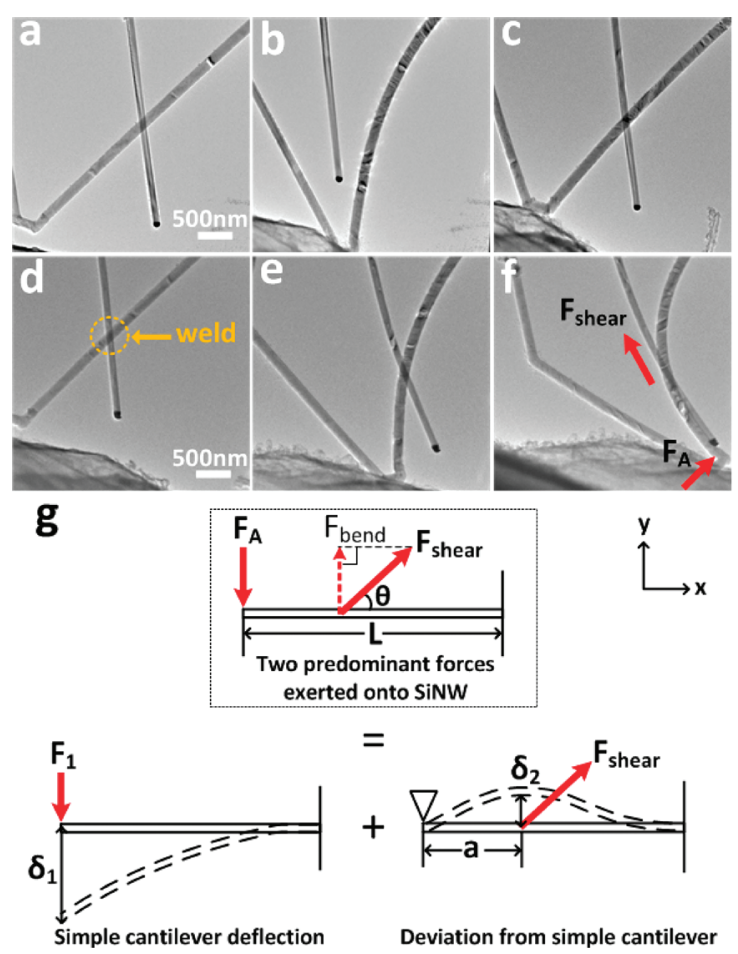

Figure 3. Determination of welding strength at the interface of crossed SiNWs after lithiation/delithiation. (a) As seen crossed nanowires. Same scale bar of $500 \mathrm{~nm}$ from $(\mathrm{a}-\mathrm{c})$. (b) Nanowires that are pulled apart to demonstrate they are grown independently. (c) Bend contours seen in both nanowires suggest they are in contact with each other. (d) Crossed nanowires after lithiation and delithiation. Same scale bar of $500 \mathrm{~nm}$ from $(\mathrm{d}-\mathrm{f})$. (e,f) Stress applied on nanowires in order to separate them. (g) The free-body-diagram (top), which depicts the total deflection of the SiNW in (f), illustrates the two predominant forces that generate the total deflection of the nanowire; $F_{\mathrm{A}}$ is the transverse force applied by nanomanipulator, and $F_{\text {shear }}$ is the shear force exerted by the weld. The total deflection is a linear superposition of two distinct deflections: $\delta_{1}$, generated by $F_{1}$ (a component of the unknown, total applied force $F_{\mathrm{A}}$ ) and $\delta_{2}$, generated by $F_{\text {shear }}$. To calculate $F_{1}$, we model the system as a simple cantilever. To calculate $F_{\text {shear }}$, we model the system as a beam fixed at one end and pinned at the tip. $F_{\text {bend }}$ is the transverse component of $F_{\text {shear }} a$ is the distance from the free end of the NW to $F_{\text {shear, }}$ and $L$ is the total length of the NW.

Figure 3a shows two pristine crystalline SiNWs, which appear to cross each other as viewed through the TEM. We note that the nanowire closest to the lithium source exhibits kinking, which is believed to be due to twinning along the growth direction. This is commonly observed among bottom-up grown SiNWs (approximately $40 \%$ of the total SiNWs), ${ }^{31,32}$ but has no contribution or observed effect on the lithiation processes in this study. In order to confirm that the crossed nanowires are not the branches of the same parent nanowire, but instead, physically distinct nanostructures, we separated one of the nanowires from the other using the nanomanipulator (Figure $3 b)$. Because they are easily separated from one another, both nanowires are observed to be separate entities rather than a single, branched, or defective nanostructure. Next, the nanowires are brought back into physical contact with each other using the piezo-controlled STM probe. Single-crystal nanowires 
that are bent exhibit a strong diffraction contrast in the form of Bragg-condition bend contours. These bend contours appear to move along the length of the nanowire as it is being strained. ${ }^{33}$ The observed motion of the bend contours in both SiNWs unambiguously confirms that they contact one another (Figure 3c). Next, the SiNWs are lithiated and delithiated (Figure 3d) using similar parameters to those used for the experiment shown in Figure 2.

We perform two tests to determine if strong bonds are formed between two SiNWs after a single lithiation/delithiation cycle. First, we attempt to bring two SiNWs into and out of contact by applying positive and negative forces, normal to the plane of the SiNW junction (see Figure S4 in Supporting Information). Prior to lithiation, the negative normal force overcomes any van der Waals bonding that occurs when the two nanowires touch. After lithiation and delithiation, application of a negative normal force no longer separates the nanowires, suggesting the presence of a strong bond. Our second test, as demonstrated in Figure $3 \mathrm{~d}-\mathrm{f}$ and described in detail as follows, allows us to assign a value to the mechanical strength of the weld. Although the strength of the weld cannot be precisely determined using our experimental data, we can estimate a lower limit to its mechanical strength by the observation that it was able to withstand the loading depicted in Figure $3 \mathrm{f}$, just prior to failure of the weld. To estimate this lower bound, we modeled the primary $\mathrm{SiNW}$ as a suspended cantilever, as shown in the free-body diagram of Figure 3g. This $\mathrm{SiNW}$ is fixed at one end at the silicon growth substrate and is fully suspended in vacuum at all other points along its axis. The deformed shape of this SiNW, as shown in Figure 3f, results from two concentrated forces, a transverse force at its free end exerted by the moving Li source $\left(F_{\mathrm{A}}\right.$ in Figure $\left.3 \mathrm{f}, \mathrm{g}\right)$, and a shear force from the weld with the secondary SiNW ( $F_{\text {shear }}$ in Figure $3 \mathrm{f}, \mathrm{g})$. The combined effect of these two forces on the deformation of the suspended SiNW is equivalent to the superposition of two decoupled configurations as illustrated in Figure $3 g$. In one configuration, the suspended $\mathrm{SiNW}$ is subject to a transverse force (labeled as $F_{1}$ ) at its free end. In another configuration, the displacement of the free end of the SiNW is fixed by a pin and the SiNW is subject to the concentrated force $F_{\text {shear }}$. The resulting deflection of the SiNW in these two configurations can be solved analytically (as detailed in Section III of Supporting Information) from which $F_{\text {shear }}$ and the shear strength can be estimated. From this analysis, we estimate the shear strength to be at least $200 \mathrm{MPa}$. Thus, our analytical model suggests that the strength of the fused $\mathrm{Si}$ region is comparable to that of stainless steel $(205 \mathrm{MPa})^{34}$ and ceramic silicon carbide $(\sim 200 \mathrm{MPa}),{ }^{35}$ thus demonstrating that a strong bond has been formed by the electrochemical cycling. To further verify the calculated strength, we also perform threedimensional nonlinear finite-element analysis (FEA) (as detailed in Section IV of Supporting Information). This analysis addresses any nonlinear effects arising from large deformations as depicted in Figure $3 \mathrm{e}, \mathrm{f}$, and it leads to an estimated shear stress at the welded region of about $308 \mathrm{MPa}$, confirming the robust strength of the weld. Similar mechanical studies were carried out on a total of five separate nanowire junctions with qualitatively similar results, confirming that the strong bonding is a reproducible feature.

The observation of strong bonding in the welded region and facile lithium transport through the SiNW interface during lithiation and delithiation can be explained as follows. Generally, as $\mathrm{Li}$ intercalates into the SiNW, the energy barrier for breaking the strongly covalent $\mathrm{Si}-\mathrm{Si}$ bonds is significantly reduced. Initially, the $\mathrm{Si}-\mathrm{Si}$ bonding energy is very high $(2.72$ $\mathrm{eV}$ ) but, according to a model put forth by Zhao et al., ${ }^{36}$ when a single $\mathrm{Si}-\mathrm{Si}$ bond is surrounded by four $\mathrm{Li}$ atoms the energy barrier for breaking the bond is reduced to a mere $0.08 \mathrm{eV}$. Consequently, $\mathrm{Si}-\mathrm{Si}$ bonds readily break, as they also tend to stretch, during $\mathrm{Li}$ intercalation. As $\mathrm{Si}-\mathrm{Si}$ bonds are broken, the dangling bonds are saturated by neighboring $\mathrm{Li}$ atoms in order to lower the system's free energy. This model may be extended to our specific study of Li-assisted welding at the interface of two SiNWs. We observe that $\mathrm{Li}-\mathrm{Si}$ bond formation occurs first at the high-energy nanowire surface before the lithiation reaction propagates inward toward the nanowire core. When the surfaces of two nanowires are directly in contact, $\mathrm{Li}$ atoms can bond simultaneously with the surface atoms on both the primary and secondary nanowires. Our results indicate that there is no additional energy barrier to diffusing to the surface of the second nanowire along this interfacial contact and reacting with this secondary surface as well. As a result, during intercalation, the surface energy of the crossed-SiNW system is reduced by forming metastable $\mathrm{Li}-\mathrm{Si}$ bonds, which bridge the two nanowires at the interface. However, during $\mathrm{Li}$ extraction this $\mathrm{Si}-\mathrm{Li}-\mathrm{Si}$ bridge is broken and there is a kinetic driving force for neighboring $\mathrm{Si}$ atoms at the interface to bond to one another. Thus, the decrease of surface energy is the kinetic driving force for welding SiNWs. Moreover, the reconstructing of surface $\mathrm{Si}-\mathrm{Si}$ bonds by Li transport across connected SiNWs makes the welding phenomenon possible at room temperature. Finally, we note that the SiNWs used in our experiments were all encompassed by a $\sim 2 \mathrm{~nm}$ layer of native $\mathrm{SiO}_{x}$, but, this $\mathrm{SiO}_{x}$ layer apparently does not complicate the $\mathrm{Si}-\mathrm{Si}$ bonding process. One possible explanation for this is that the $\mathrm{SiO}_{x}$ may be reduced into $\mathrm{Si}, \mathrm{Li}_{2} \mathrm{O}$ and $\mathrm{Li}$-containing silicates upon $\mathrm{Li}^{+}$insertion, of which the latter two may also be serving to enhance the strength of the welded junction. ${ }^{37-39}$ A second possibility is that the volume expansion of the SiNW during lithium insertion may also break the $\mathrm{SiO}_{x}$ layer, thereby enabling direct contact between the interfacial host atoms. ${ }^{24,26,40}$ This explains the $\mathrm{Li}-\mathrm{Si}$ bonding and corresponding phase changes at the interface of the crossed SiNW system.

To summarize, we have directly observed electrochemical welding of cross-contacted SiNWs at ambient temperatures during lithiation and delithiation by in situ TEM studies. By comparing the incremental expansion and contraction of the SiNWs that are in direct contact with the $\mathrm{Li}$ source to those that are not, we have demonstrated that there is facile $\mathrm{Li}$ transport between two SiNW interfaces. We also estimate the minimum mechanical shear strength of the interfacial weld between two SiNWs to be $\sim 200 \mathrm{MPa}$, indicating that this welding process generates strong interfacial bonds. The inherently high capacity of silicon, combined with facile $\mathrm{Li}$ diffusion between surface-contacted $\mathrm{Si}$ nanostructures, may enable the self-assembly of an interconnected network for Si nanowires in anodes, leading to high energy density lithium ion batteries with improved cycling stability. We suggest that this welding phenomenon may also serve as a self-healing mechanism in Li-ion battery electrodes. It is well-known that during cycling SiNWs may lose electrical contact with the electrode support, but our experimental results indicate that such detached nanowires may still actively participate in $\mathrm{Li}$ storage if they remain part of a robust contacted network, formed during the first lithiation cycle. ${ }^{41}$ Furthermore, the welding and self-healing processes that we have observed in 
SiNWs may equally apply to other alloy-based anode materials, such as germanium and tin.

\section{ASSOCIATED CONTENT}

\section{S Supporting Information}

Sample preparation technique (Figure S1), lithiation and delithiation behavior in another set of physically contacted crossed SiNWs (Figure S2), and detailed description of the simplified analytical model and nonlinear finite element analysis for shear strength at its welded interface. This material is available free of charge via the Internet at http://pubs.acs.org.

\section{AUTHOR INFORMATION}

\section{Corresponding Author}

*E-mail: (C.W.) cswang@umd.edu; (J.C.) cumings@umd.edu.

\section{Notes}

The authors declare no competing financial interest.

\section{ACKNOWLEDGMENTS}

This work was supported as part of the Science of Nanostructures for Electrical Energy Storage, an Energy Frontier Research Center funded by the U.S. Department of Energy, Office of Science, Office of Basic Energy Sciences under Award Number DESC0001160. The support of the Maryland NanoCenter and shared experimental facilities support from the NSF MRSEC under Grant DMR 05-20471 are also gratefully acknowledged. T.L. and Z.J. acknowledge the support of NSF under Grants 0856540 and 0928278 . Work at Los Alamos National Laboratory was performed under U.S. Department of Energy contract DE-AC52-06NA25396. This research was performed, in part, at the Center for Integrated Nanotechnologies (CINT), a U.S. Department of Energy, Office of Basic Energy Sciences user facility

\section{REFERENCES}

(1) Aricò, A. S.; Bruce, P.; Scrosati, B.; Tarascon, J. Schalkwijk. Nanostructured materials for advanced energy conversion and storage devices. Nature 2005, 4, 366-377.

(2) Park, C.; Kim, J.; Kim, H.; Sohn, H. Li-alloy based anode materials for Li secondary batteries. Chem. Soc. Rev. 2010, 39, 31153141.

(3) Zhang, W. Lithium insertion/extraction mechanism in alloy anodes for lithium-ion batteries. J. Power Sources 2011, 196, 13-24.

(4) Obravac, M. N.; Christensen, L. Structural changes in silicon anodes during lithium insertion/extraction. Electrochem. Solid-State Lett. 2004, 7 (5), A93-A96.

(5) Kasavajjula, U.; Wang, C.; Appleby, A. J. Nano- and bulk- siliconbased insertion anodes for lithium- ion secondary cells. J. Power Sources 2007, 163, 1003-1039.

(6) Chan, C. K.; Peng, H.; Liu, G.; Mcllwrath, K.; Zhang, X. F.; Huggins, R. A.; Cui, Y. High-performance lithium battery anodes using silicon nanowires. Nat. Nanotechnol. 2008, 3, 31.

(7) Chandrasekaran, R.; Megasinki, A.; Yushin, G.; Fuller, T. F. Analysis of lithium insertion/deinsertion in a silicon electrode particle at room temperature. J. Electrochem. Soc. 2010, 157 (10), A1139A1151.

(8) Christensen, J.; Newman, J. Stress generation and fracture in lithium insertion materials. J. Solid State Electrochem. 2006, 10 (5), 293-319.

(9) Kalnaus, S.; Rhodes, K.; Daniel, C. A study of lithium ion intercalation induced fracture of silicon particles used as anode materials in Li-ion battery. J. Power Sources 2011, 196, 8116-8124.

(10) Shenoy, V. B.; Johari, P; Qi, Y. Elastic softening of amorphous and crystalline $\mathrm{Li}-\mathrm{Si}$ phases with increasing $\mathrm{Li}$ concentration: A firstprinciple study. J. Power Sources 2010, 195, 6825-6830.
(11) Sethuraman, V. A.; Chon, M. J.; Shimshak, M.; Srinivasan, V.; Guduru, P. R. In-situ measurements of stress evolution in silicon thin films during electrochemical lithiation and delithiation. J. Power Sources 2010, 195, 5062-5066.

(12) Sethuraman, V. A.; Srinivasan, V.; Bower, A. F.; Guduru, P. R. In situ measurements of stress-potential coupling in lithiated silicon. J. Electrochem. Soc. 2010, 157 (11), A1253-A1262.

(13) Takamura, T.; Ohara, S; Uehara, M.; Suzuki, J.; Sekine, K. A vacuum deposited Si film having a Li extraction capacity over 2000 $\mathrm{mAh} / \mathrm{g}$ with a long cycle life. J. Power Sources 2004, 129, 96-100.

(14) Kim, H.; Han, B.; Chou, J.; Cho, J. Three-dimensional porous silicon particles for use in high performance lithium secondary batteries. Angew. Chem. 2008, 47, 10151-10154.

(15) Park, M.; Kim, M. G.; Joo, J.; Kim, K.; Kim, J.; Ahn, S.; Cui, Y.; Cho, J. Silicon Nanotube Battery Anodes. Nano Lett. 2009, 9 (11), 3844-3847.

(16) Song, T.; Xia, J. L.; Lee, J. H.; Lee, D. H.; Kwon, M. S.; Choi, J. M.; Wu, J.; Dou, S. K.; Chang, H.; Park, W. I.; Zang, D. S.; Kim, H.; Huang, Y. G.; Hwang, K. C.; Rogers, J. A.; Paik, U. Arrays of Sealed Silicon Nanotube As Anodes for Lithium Ion Batteries. Nano Lett. 2010, 10, 1710.

(17) Szczeh, J. R.; Jin, S. Nanostructured silicon for high capacity lithium battery anodes. Energy Environ Sci. 2011, 4, 56-72.

(18) Lee, K. T.; J. Cho, J. Roles of nanosize in lithium reactive nanomaterials for lithium ion batteries. Nano Today 2011, 6, 28-41.

(19) Ryu, I.; Choi, J. W.; Cui, Y.; Nix, W. D. Size-dependent fracture of Si nanowire battery anodes. J. Mech. Phys. Solids. 2011, 59, 17171730.

(20) Li, J.; Dozier, A. K.; Li, Y.; Yang, F.; Cheng, Y. Crack pattern formation in thin films lithium-ion battery electrodes. J. Electrochem. Soc. 2011, 158 (6), A689-A694.

(21) Goldman, J. L.; Long, B. R.; Gewirth, A. A.; Nuzzo, R. G. Strain anisotrophies and self-limiting capacities in single-crystalline 3D silicon microstructures: Models for high energy density lithium-ion battery anodes. Adv. Funt. Mater. 2011, 21, 2412-2422.

(22) Liu, X. H.; Zheng, H.; Zhong, L.; Huang, S.; Karki, K.; Zhang, L. Q.; Liu, Y.; Kushima, A.; Liang, W. T.; Wang, J. W.; Cho, J.; Epstein, E.; Dayeh, S. A.; Picraux, S. T.; Zhu, T.; Li, J.; Sullivan, J. P.; Cumings, J.; Wang, C.; Mao, S. X.; Ye, Z. Z.; Zhang, S.; Huang, J. Y. Anisotropic Swelling and Fracture of Silicon Nanowires during Lithiation. Nano Lett. 2011, 11, 3312-3318.

(23) Courtney, I. A.; McKinnon, W. R.; Dahn, J. R. On the aggregation of tin in $\mathrm{SnO}$ composite glasses caused by the reversible reaction with lithium. J. Electrochem. Soc. 1999, 146, 59-68.

(24) Li, H.; Huang, X.; Chen, L.; Zhou, G.; Zhang, Z.; Yu, D.; Mo, Y. J.; Pei, N. The crystal structural evolution of nano-Si anode caused by lithium insertion and extraction at room temperature. Solid State Ionics 2000, 135, 181-191.

(25) Shi, L.; Li, H.; Wang, Z.; Huang, X.; Chen, L. Nano-SnSb alloy deposited on MCMB as an anode material for lithium ion batteries. J. Mater. Chem. 2001, 11, 1502-1505.

(26) Li, H.; Shi, L.; Lu, W.; Huang, X.; Chen, L. Studies on capacity loss and capacity fading of nanosized $\mathrm{SnSb}$ alloy for Li-ion batteries. J. Electrochem. Soc. 2001, 148, A915-A922.

(27) Ryu, J. H.; Kim, J. W.; Sung, Y.; Oh, S. M. Failure modes of silicon powder negative electrode in lithium secondary batteries. Electrochem. Solid-State Lett. 2004, 7, A306-A309.

(28) Liu, Y.; Zheng, H.; Liu, X. H.; Huang, S.; Zhu, T.; Wang, J.; Kushima, A.; Hudak, N. S.; Huang, X.; Zhang, S.; Mao, S. X.; Qian, X.; Li, J.; Huang, J. Y. Lithiation-induced embrittlement of multiwalled carbon nanotubes. ACS Nano 2011, 5 (9), 7245-7253.

(29) Liu, X. H.; Huang, S.; Picraux, S. T.; Li, J.; Zhu, T.; Huang, J. Y. Reversible nanopore formation in Ge nanowires during lithiationdelithiation cycling: An in situ transmission electron microscope study. Nano Lett. 2011, 11 (9), 3991-3997.

(30) Kharkats, Y. I. Transition to a state of superionic conduction induced by an electric field. Sov. Phys. Solid State 1981, 23, 12831284. 
(31) Cayron, C.; Hertog, M. D.; Latu-Romain, L.; Mouchet, C.; Secouard, C.; Rouviere, J.; Rouviere, E.; Simonato, J. Odd electron diffraction patterns in silicon nanowires and silicon thin films explained by microtwins and nanotwins. J. Appl. Crystallogr. 2009, $42,242-252$.

(32) Dayeh, A. A.; Wang, J.; Li, N.; Huang, J. Y.; Gin, A. V.; Picraux, S. T. Growth, Defect Formation, and Morphology Control of Germanium-Silicon Semiconductor Nanowire Heterostructures. Nano Lett. 2011, 11, 4200-4206.

(33) Williams, D. B.; Carter, C. B. Transmission Electron Microscopy A Textbook for Materials Science, 2nd ed.; Springer: New York, 2009.

(34) Shackelford, J. F.; Alexander, W. CRC Materials Science and Engineering Handbook, 3rd ed.; CRC Press: Boca Raton FL, 2001; p 628.

(35) Shackelford, J. F.; Alexander, W. CRC Materials Science and Engineering Handbook, 3rd ed.; CRC Press: Boca Raton FL, 2001; p 1587

(36) Zhao, K.; Wang, W. L.; Gregoire, J.; Pharr, M.; Suo, Z.; Vlassak, J. J.; Kaxiras, E. Lithium-Assisted Plastic Deformation of Silicon Electrodes in Lithium-Ion Batteries: A First Theoretical Study. Nano Lett. 2011, 11, 2962-2967.

(37) Sun, Q.; Zhang, B.; Fu, Z. Lithium electrochemistry of $\mathrm{SiO}_{2}$ thin film electrode for lithium-ion batteries. Appl. Surf. Sci. 2008, 254, 3774-3779.

(38) Guo, B.; Shu, J.; Wang, Z.; Yang, H.; Shi, L.; Liu, Y.; Chen, L. Electrochemical reduction of nano- $\mathrm{SiO}_{2}$ in hard carbon as anode material for lithium ion batteries. Electrochem. Commun. 2008, 10, $1876-1878$

(39) Yamada, Y.; Iriyama, Y.; Abe, T.; Ogumi, Z. J. Kinetics of electrochemical insertion and extraction of lithium ion at $\mathrm{SiO}$. Electrochem. Soc. 2010, 157, A26-A30.

(40) Li, H.; Shi, L.; Wang, Q.; Chen, L.; Huang, X. Nano-alloy anode for lithium ion batteries. Solid State Ionics 2002, 148, 247-258.

(41) Nguyen, H. T.; Yao, F.; Zamfir, M. R.; Biswas, C.; So, K. P.; Lee, Y. H.; Kim, S. M.; Cha, S. N.; Kim, J. M.; Pribat, D. Highly interconnected $\mathrm{Si}$ nanowires for improved stability Li-ion battery anodes. Adv. Energy Mater. 2011, 1 (6), 1154-1161. 


\section{Supporting Information}

Lithium-assisted electrochemical welding in silicon nanowire battery electrodes

Khim Karki ${ }^{\dagger}$, Eric Epstein ${ }^{\dagger}$, Jeong-Hyun Cho ${ }^{\S}$, Zheng Jia ", Teng Li $"$, S. Tom Picraux ${ }^{\S}$, Chunsheng Wang * $\ddagger$, and John Cumings ${ }^{*}{ }^{\dagger}$

${ }^{\dagger}$ Department of Materials Science and Engineering, University of Maryland, College Park, MD 20742 USA

${ }^{\S}$ Center for Integrated Nanotechnologies, Los Alamos National Laboratory, Los Alamos, NM 87545 USA

"Department of Mechanical Engineering, University of Maryland, College Park, MD 20742 USA

${ }^{\ddagger}$ Department of Chemical and Biomolecular Engineering, University of Maryland, College Park, MD 20742 USA

\section{Sample Preparation}

The silicon nanowires (SiNWs) used in this study were grown from the (111) Si surface at the edge of a window in a silicon nitride (SiN) TEM membrane (SPI supplies) using the bottom-up vapor-liquid-solid (VLS) technique. The dimensions of the TEM-grid window are $500 \mu \mathrm{m} \times 500 \mu \mathrm{m}$ with a $100 \mathrm{~nm}$ thick silicon nitride membrane. In order to break the membrane, the TEM grid was sonicated for 5 seconds in isopropanol. Gold colloids (100 nm diameter) were then dispersed around the edge of the TEM window. The samples were loaded in a low-pressure CVD reactor, and the temperature was set to $490^{\circ} \mathrm{C}$ in order for the gold colloids to form a eutectic mixture with Si. Silane ( $50 \% \mathrm{SiH}_{4}$ in $\mathrm{H}_{2}$ ) and phosphine (100 ppm $\mathrm{PH}_{3}$ in $\mathrm{H}_{2}$ ) were introduced into the chamber simultaneously with the flow rate of $250 \mathrm{sccm}$ and $100 \mathrm{sccm}$ respectively with a chamber pressure of 3 Torr. The growth time was 10 minutes with the resulting nanowires $\sim 10 \mu \mathrm{m}$ in length, $100-150 \mathrm{~nm}$ in diameter, and moderately $\mathrm{n}$-doped.

\section{Supporting Figures}

Figure S1: SEM of SiNWs grown on the edges of a silicon nitride TEM window. The inset shows a single nanowire atop the beveled (111) Si substrate.

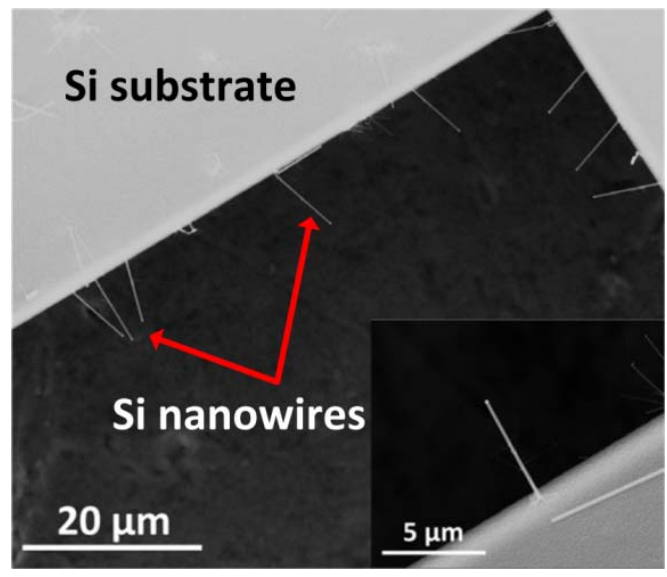



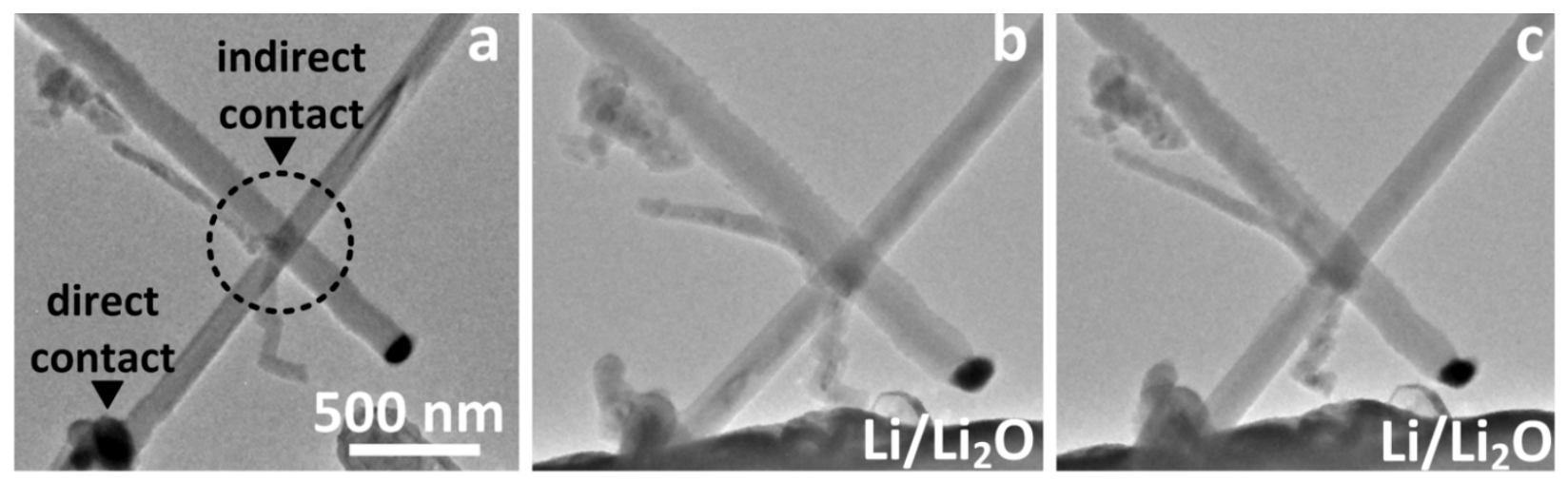

Figure S2 A: Lithiation and delithiation of a second set of physically-contacted SiNWs. a) Pristine SiNWs brought in contact. b) Swelling of SiNWs observed after lithiation. A bias of -3V vs. lithium metal is applied. c) Contraction of SiNWs after delithiation. A bias of $+3 \mathrm{~V}$ vs. lithium metal is applied.

Figure S3 >: Mechanical separation of welded pair of SiNWs from Figure S2. a) SiNWs after one lithiation and delithiation. b) SiNWs pulled away with a transverse force. c) SiNW broken off from the substrate as a result of an applied force is seen supported only by the welded support.
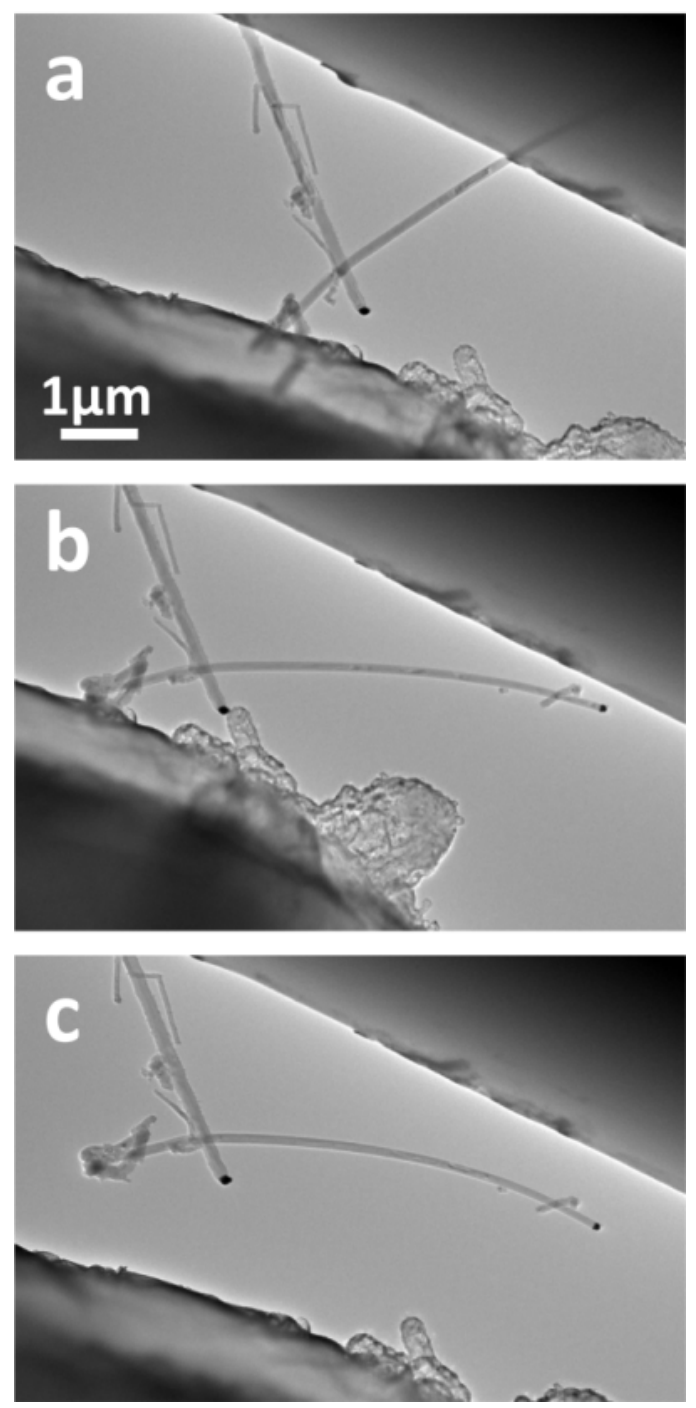

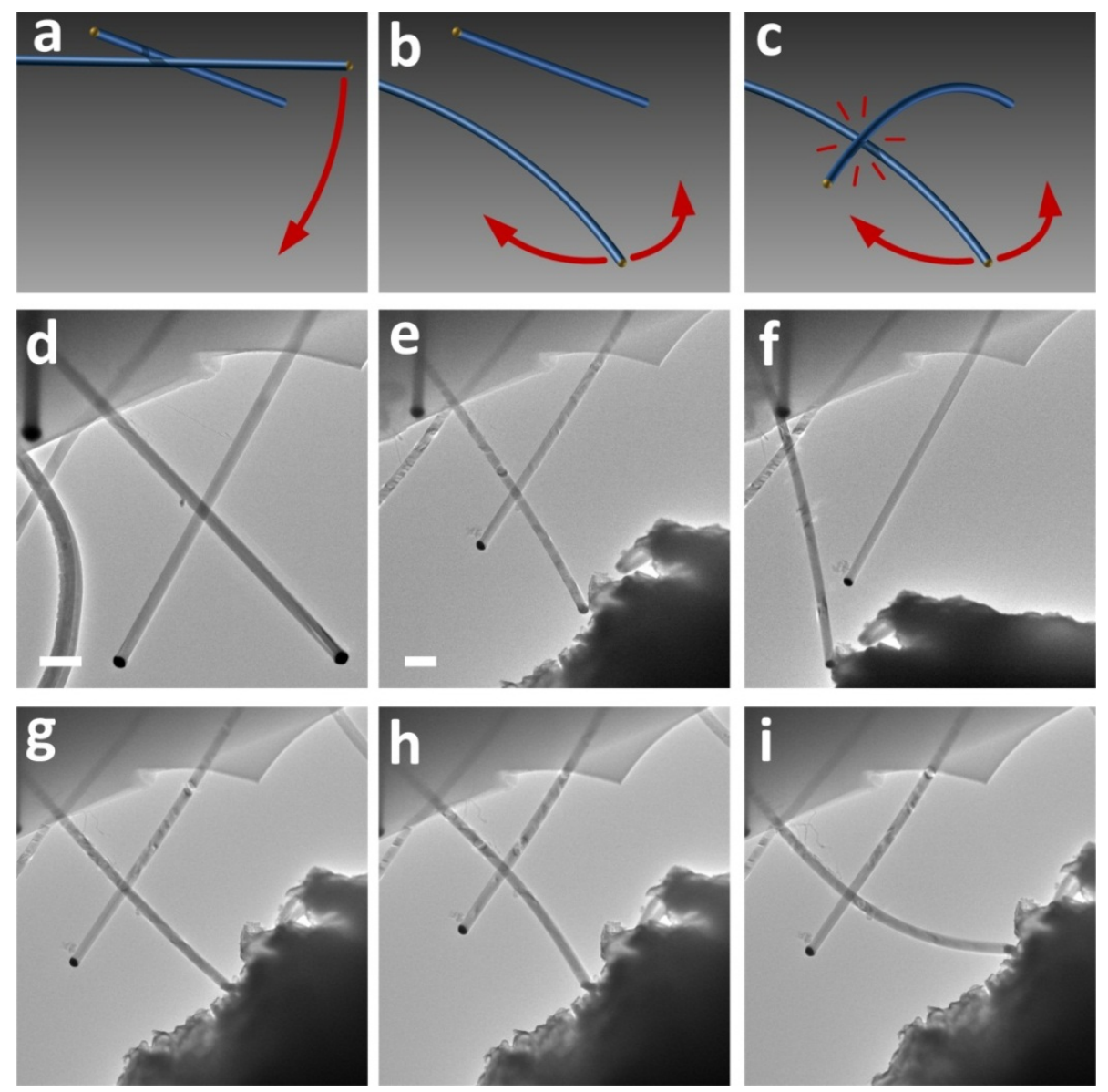

Figure S4: Mechanical testing of a third set of physically-contacted SiNWs before and after lithiation and delithiation. a), b), and c) show schematic 3D representations of mechanical tests. In a), the bottom wire of the two contacting crossed SiNWs can be bent downward, providing a negative load to the bonded interface. In b), the negative force separates the SiNWs, revealing a weak Van der Waals bond, prior to the welding. This can be confirmed by lateral bending of the lower SiNW, which has no influence on the mechanical state of the upper SiNW, when they are not in contact. In c), the negative force produces a downward deflection of the upper SiNW, revealing that a strong bond has been formed by the welding process. The strength of the weld can be further confirmed by lateral bending of the lower SiNW, which reveals the weld junction as an anchor point. d) A TEM image of a pair of pristine as-grown SiNWs, which are not in contact. Scale bar of $500 \mathrm{~nm}$. The SiNWs can reversibly be brought into contact (Van der Waals bond), as shown in e), and separated, as shown in f). Same scale bar of $500 \mathrm{~nm}(\mathrm{e}-\mathrm{i})$. The contacted, lithiated, and delithiated pair of wires is shown in g), after the welding has been formed. As depicted in schematic $\mathrm{c}$ ), the strength of the weld can be confirmed by negative loading of the welded junction and subsequent lateral loading of the contacting SiNW, as shown in $\mathrm{h}$ ) and i), respectively. 


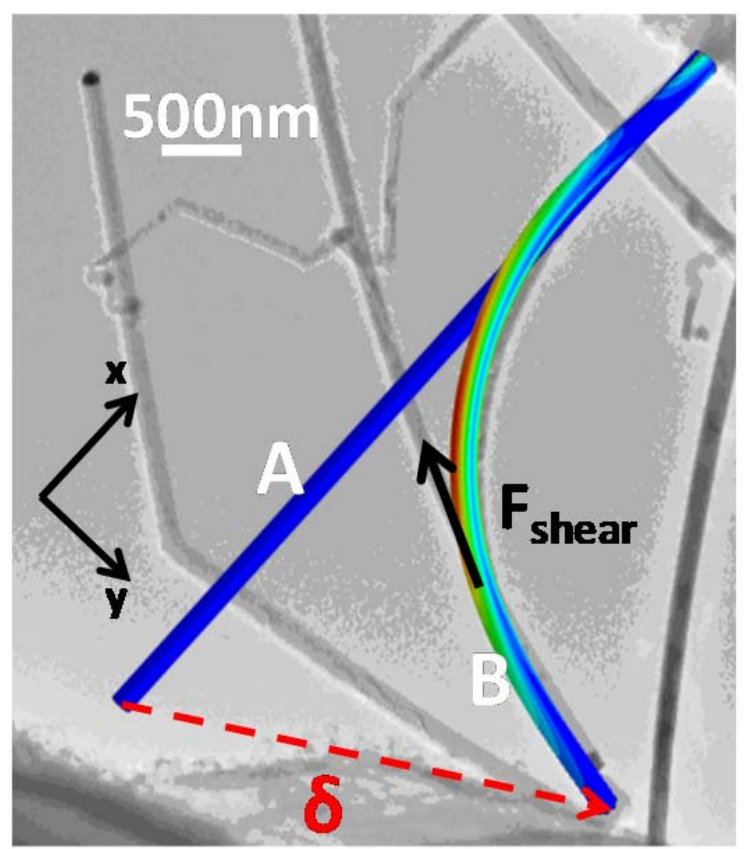

\section{A: Un-deformedSiNW \\ B: Deformed shape of SiNW from FEA simulation}

Figure S5: The undeformed and deformed shapes of SiNW from the best-fit FEA simulation are plotted over the in situ TEM image of the deformed SiNW (same as Figure 3f). The color shades represent von Mises stress levels in the SiNW.

\section{A simplified analytical model for calculation of shear strength at the welded interface of the crossed SiNWs}

The total deflection of the SiNW shown in Figure $3 \mathrm{~g}$ in the transverse direction is a superposition of the

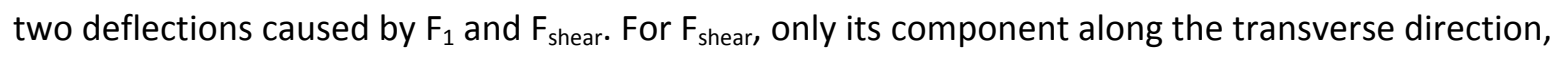
labeled as $\mathrm{F}_{\text {bend, }}$, contributes to the deflection of the SiNW. To determine the shear strength at the welded interface, we first estimate $F_{\text {bend. }}$. The equations that follow are derived using the double integration method on the governing equation for the deformation of an elastic beam: $\frac{d^{2} y(x)}{d x^{2}}=\frac{\mathrm{M}}{\mathrm{EI}^{\prime}}$ where $y(x)$ is the deflection of the beam as a function of location $x, M$ is the bending moment of the beam with respect to its neutral axis, $I$ is the area moment of inertia of the beam $\left(=2.9 \times 10^{-29} \mathrm{~m}^{4}\right.$, using SiNW radius of $78 \mathrm{~nm}$ and assuming a circular cross-section), and $\mathrm{E}$ is the elastic modulus of the beam (taken to be $68.6 \mathrm{GPa}$, representative for amorphous Si [S1-S3]). In all calculations, the origin is taken to 
be the tip of the SiNW. The unknown value of $F_{A}$ is a superposition of two forces: a reaction force from the pin in the bottom right of Figure $3 g$ and $F_{1}$, the force that would be required to produce deflection $\delta_{1}$ in the absence of $F_{\text {bend. }}$. We compute $F_{1}$ according to the following equation,

$$
\mathrm{F}_{1}=\frac{3 \mathrm{EI} \delta_{1}}{\mathrm{~L}^{3}} \cong 7.7 * 10^{-6} \mathrm{~N}
$$

Here, $L$ is the length of the SiNW $(5780 \mathrm{~nm})$ and $\delta_{1}=2480 \mathrm{~nm}$ is the transverse displacement from the neutral axis of the undeflected SiNW (Figure 3a) to the neutral axis of the fully deflected SiNW (Figure 3f). From this estimate of $F_{1}$, we calculate the deflection near the intersection of two welded SiNW (labeled by $x_{c}$ from the origin) as it should be, if $F_{1}$ were the only force acting on the SiNW,

$$
\delta_{\text {calc }}=\frac{\mathrm{F}_{1}}{6 \mathrm{EI}}\left(2 \mathrm{~L}^{3}-3 \mathrm{~L}^{2} \mathrm{x}_{\mathrm{c}}+\mathrm{x}_{\mathrm{c}}{ }^{3}\right) \cong 580 \mathrm{~nm} .
$$

However, $\mathrm{F}_{\text {bend }}$ from the secondary SiNW causes the observed deflection at $\mathrm{x}_{\mathrm{c}}$ to deviate from the abovecalculated value by an amount, $\delta_{2}=430 \mathrm{~nm}$, which is directly measured from microscope images near the welded intersection (see Figure 3 ). Thus, $\delta_{2}$ is the additional deflection induced by $F_{\text {bend }}$ at $x_{c}$, as illustrated in the bottom right configuration in Figure $3 g$. The force $F_{\text {bend }}$ can be calculated from $\delta_{2}$ according to the following equation,

$$
\mathrm{F}_{\text {bend }}=\frac{12 \mathrm{EIL}^{3} \delta_{2}}{\mathrm{a}\left(\mathrm{L}-\mathrm{x}_{\mathrm{C}}\right)^{2}\left[3 \mathrm{~L}\left(\mathrm{a}^{2}-\mathrm{L}^{2}\right)+\left(\mathrm{L}-\mathrm{x}_{\mathrm{c}}\right)\left(3 \mathrm{~L}^{2}-\mathrm{a}^{2}\right)\right]} \cong 6.6 * 10^{-7} \mathrm{~N}
$$

where $a$ is the distance from the pinned end of the SiNW to the point where $F_{\text {bend }}$ is applied [S4]. Using the included angle between $F_{\text {bend }}$ and $F_{\text {shear }}\left(24^{\circ}\right)$, the value of $F_{\text {shear }}$ can then be estimated. The shear strength at the welded junction (the stress prior to failure) can be then calculated by dividing $F_{\text {shear }}$ by the cross-sectional area of the welded junction. To estimate an upper bound of the welded area, we can use the total cross-section of the nanowires as it appears in projection in a TEM image, yielding a lower bound on the shear strength of $140 \mathrm{MPa}$. However, two cylindrical nanowires would be unlikely to form a weld with such a large cross-sectional area. To improve the estimate, we can use a geometrical model, incorporating space-filling during the lithiation expansion followed by uniform contraction, to obtain a more accurate estimate of the cross-sectional area. From this more realistic geometrical model, we obtain a shear strength of approximately $200 \mathrm{MPa}$. We note that the analytical model used here assumes no deflections parallel to the direction of the imaging electron beam, since all 2D images are obtained in projection. However, the manipulation piezo causing the deflections is actuated purely in the plane of the image, and thus any out-of-plane deflections are expected to introduce only minimal 
correction factors. We note that the above analysis assumes no bending moment is introduced due to the torsional loading of the weld itself. If such a load were included, then the inhomogeneous stress within the weld would result in an increased estimate of the strength of the weld material. We further note that the geometry of the lithiated nanowires can be anisotropic, as described in previous studies [S5-S7]. However, we neglect this effect because the degree of anisotropy is dependent upon the degree of lithiation. The welded SiNWs used in our study are not highly anisotropic because the SiNWs are not fully lithiated, and thus the above model is expected to be a good approximation.

\section{Estimation of shear strength at the welded interface via nonlinear finite element analysis of the large deformation of SiNW}

The simplified analytical model delineated in Section III guarantees a good estimate of the shear strength at the welded interface of the crossed SiNWs only when the deflection of the SiNW is not significantly large. To further quantify the effect of the large deflection of the SiNW in our in situ experiment as shown in Figure 3, we next carry out a three-dimensional finite element analysis (FEA) accounting for the nonlinear geometric effect of the large deformation of the SiNW, using the software package, ABAQUS v6.10. The FEA model considers a similar suspended cantilever SiNW as illustrated in Figure 3g. The free end of the SiNW is subject to a displacement $\delta$ with two components of $\delta_{\mathrm{x}}=2150 \mathrm{~nm}$ and $\delta_{\mathrm{y}}=3020 \mathrm{~nm}$, which are measured by direct comparison of the in situ TEM images of the undeformed and deformed SiNW. The effect of the secondary SiNW on the deformation of the suspended cantilever SiNW is introduced by a shear force $F_{\text {shear }}$ acting at the welded intersection along the axial direction of the secondary SiNW. In our FEA simulation model, the SiNW is $5780 \mathrm{~nm}$ long and its crosssection is assumed to be circular with a radius of $r=78 \mathrm{~nm}$. The SiNW is modeled as linear elastic material with Young's Modulus and Poisson's ratio to be 68.6GPa and 0.3, respectively. The SiNW is meshed with three-dimensional-stress eight-node linear brick elements (C3D8R) with sizes about 0.2r. Nonlinear geometry option is turned on in the simulations to account for the large deformation of the SiNW. The value of $F_{\text {shear }}$ is varied in simulations until the deformed shape of the SiNW reaches the best fit to the deformed SiNW as observed in the TEM image. Figure S5 plots the undeformed and deformed SiNW from the best-fit FEA simulation overlapping the TEM image of the deformed SiNW (same as Figure 3f). The best-fit FEA simulation gives $F_{\text {shear }}=2.5 \times 10^{-6} \mathrm{~N}$. Using the same method to estimate the crosssectional area of the welded junction as described in Section III, the shear strength is estimated to be $308 \mathrm{MPa}$, somewhat larger than that estimated by the simplified analytical model. The difference between these two estimates may be attributed to nonlinear geometric effects of the large deformation 
of the SiNW. However, to be conservative, the value of $200 \mathrm{MPa}$ estimated by the analytical model may be taken to be the conclusion of our studies.

\section{Supporting References}

[S1] Allred, C.; Yuan, X.; Bazant, M.; Hobbs, L. Elastic constants of defected and amorphous silicon with the environment-dependent interatomic potential. Phys. Rev. B. 2004, 70, 134113.

[S2] Ni, H.; Li, X.; Gao, H. Elastic modulus of amorphous $\mathrm{SiO}_{2}$ nanowires. Appl. Phys. Lett. 2006, 88, 043108.

[S3] Sohn, Y.; Park, J.; Yoon, G.; Song,J.; Jee, S.; Lee, J.; Na,S.; Kwon, T.; Eom, K. Mechanical Properties of Silicon Nanowires. Nanoscale Res. Lett. 2010, 5, 211-216.

[S4] Patnaik, S.N.; Hopkins, D.A. Strength of Materials A New Unified Theory for the $21^{\text {st }}$ Century, First Edition, 2004, p.697, Elsevier, MA.

[S5] Goldman, J.L.; Long,B.R.; Gewirth,A.A.; Nuzzo, R.G. Strain anisotrophies and self-limiting capacities in single-crystalline 3D silicon microstructures: Models for high energy density lithium-ion battery anodes. Adv. Funt. Mater. 2011, 21, 2412-2422.

[S6] Lee, S.K.; McDowell, M.T.; Choi, J.W.; Cui, Y. Anomalous shape changes of silicon nanopillars by electrochemical lithiation. Nano Lett. 2011, 11, 3034-3039.

[S7] Liu, X.H.; Zheng, H.; Zhong, L.; Huang, S.; Karki, K.; Zhang, L.Q.; Liu, Y.; Kushima, A.; Liang, W.T.; Wang, J.W.; Cho, J.; Epstein, E.; Dayeh, S.A.; Picraux, S.T.; Zhu, T.; Li, J.; Sullivan, J.P.; Cumings, J.; Wang, C.; Mao, S.X.; Ye, Z.Z.; Zhang, S.; Huang, J.Y. Anisotropic swelling and fracture of silicon nanowires during lithiation. Nano Lett. 2011, 11, 3312-3318. 